\title{
Traduire
}

Revue française de la traduction

\section{Vivre avec un traducteur indépendant : mode d'emploi}

\section{Céline Petit}

\section{Q OpenEdition}

\section{Journals}

\section{Édition électronique}

URL : http://journals.openedition.org/traduire/186

DOI : $10.4000 /$ traduire.186

ISSN : 2272-9992

\section{Éditeur}

Société française des traducteurs

\section{Édition imprimée}

Date de publication : 1 juin 2011

Pagination : 50-53

ISSN : 0395-773X

\section{Référence électronique}

Céline Petit, « Vivre avec un traducteur indépendant : mode d'emploi », Traduire [En ligne], 224 | 2011,

mis en ligne le 03 février 2014, consulté le 19 avril 2019. URL : http://journals.openedition.org/ traduire/186 ; DOI : 10.4000/traduire.186 


\section{Vivre avec un traducteur indépendant : mode d'emploi}

\section{Céline Petit}

Vous avez croisé sur votre chemin un traducteur indépendant et vous avez décidé de vivre avec. Époux, épouses, amoureux, amoureuses, partenaires de PACS, c'est à vous que je m'adresse. Voici un petit guide pour mieux comprendre le mode de vie et les besoins de l'élu de votre cœur.

Tout d'abord, notez donc que cet article traitera seulement du traducteur indépendant. Contrairement à son homologue salarié, l'indépendant est un loup constamment à l'affût d'une opportunité de travail, mais nous y reviendrons...

\section{Travail}

Quand vous partez au travail, il est assis à son bureau devant son ordinateur. Quand vous revenez du travail, il est toujours assis devant son ordinateur. Alors que vous avez enchaîné réunions, travail et déjeuner d'affaires, votre conjoint vous donne l'impression que le temps s'est arrêté chez vous. Ce n'est pas le cas. Seulement, il faut savoir que le traducteur dispose d'une étonnante capacité à passer de nombreuses heures dans la même position. D'ailleurs, son environnement est complètement optimisé en fonction de ses besoins : clavier et souris superergonomiques, écran géant configuré de manière à ne pas fatiguer les yeux, fauteuil permettant de garder une bonne posture, ordinateur puissant et suréquipé... Le traducteur a su aménager sa tanière pour y passer de longues heures. Et justement comment les occupe-t-il ?

Une fois que vous avez fermé la porte, votre cher et tendre va accomplir le premier geste de sa journée de travail : faire du thé/du café (rayez la mention inutile). Ensuite, sa fidèle tasse près de lui, il s'assoit à son bureau, dont l'équipement ne laisse rien au hasard. Observons son milieu : la chaise sur laquelle il s'assoit a fait l'objet d'une longue réflexion. Si, si, rappelez-vous l'heure entière que vous avez passée chez Ikea à tester les différents fauteuils, leur maintien, leur ergonomie, le moelleux de l'assise... pour finalement opter pour un siège dans lequel 
votre conjoint pourra passer des journées assis sans craindre pour ses lombaires ou ses cervicales. II allume son ordinateur et ses deux écrans (un pour la mémoire de traduction, l'autre pour Internet), saisit sa souris spécialement prévue pour lui éviter un syndrome du canal carpien et commence par lire ses e-mails, puis passe en revue ses abonnements RSS, ses journaux favoris, sa timeline Twitter, etc. Cela peut paraître paradoxal, mais pour quelqu'un qui sort peu de chez lui, le traducteur est souvent très bien informé de ce qui se passe à l'extérieur. Ne vous y trompez pas! Ce n'est pas parce qu'elle est capable de vous raconter heure par heure le sommet de Davos ou la négociation de la loi des finances 2011 que la prunelle de vos yeux n'a rien fait de sa journée, bien au contraire. Le traducteur est un individu multitâche capable d'écouter de la musique, de lire les mises à jour de son compte Twitter, tout en négociant son prochain contrat et en avançant dans sa traduction en cours, sa tasse caféinée à la main. Une simple question d'habitude !

II peut arriver que vous l'entendiez parler tout seul pendant longtemps. Pas de panique, n'appelez pas l'hôpital psychiatrique le plus proche : pour protéger ses mains et ses poignets, le traducteur a acquis et dompté un logiciel de dictée qui lui permet de travailler efficacement et sans risquer une entorse des doigts !

Parfois, l'envie de voir de nouvelles têtes l'amène à sortir de chez lui. Quitter son ordinateur et prendre le risque de rater une commande ? Pas de problème, votre compagnon a dans sa poche un smartphone, prolongement de son ordinateur qui lui permet d'être joignable par tous les moyens et où qu'il soit.

Un travailleur forcené, le traducteur? Heureusement, non. II a également des loisirs et une vie sociale!

\section{Loisirs}

Si vous ne deviez retenir qu'une chose sur ce spécimen qu'est le traducteur indépendant, c'est que c'est une personne avide de culture. Quoi de plus normal, quand on passe l'essentiel de sa journée à se documenter pour mieux traduire ? Ainsi, même dans ses loisirs, le traducteur a tendance à être un geek. Qu'il pratique le volley-ball, la danse orientale, qu'il joue au backgammon ou se passionne pour le scrapbooking, le traducteur s'est documenté sur l'activité qui l'occupe. L'amateur de cuisine sera capable de vous donner la date à laquelle est sorti le premier robot Kenwood, le skieur patenté est naturellement au courant des palmarès de toutes les compétitions des cinq dernières années, et je ne vous parle même pas du cinéphile ! Le pire, c'est peut-être de voir avec quelle facilité l'astre de votre vie a réussi à s'approprier ces connaissances, comme si c'était une évidence : " ben quoi, tu ne savais pas que le mascara c'était de la poudre d'antimoine au départ ? " dira l'adepte de cosmétiques, prête à enseigner l'histoire du maquillage aux vendeuses de Sephora. 


\section{Vie sociale}

Fort heureusement, le traducteur a quand même une vie sociale, ou plutôt même deux. D'abord, il y a vos proches, les amis et la famille du couple. Si ce sont des connaissances de longue date, elles sont souvent habituées aux comportements étranges du traducteur et savent qu'il est capable de raconter l'histoire de la galette des Rois dans les différentes régions de France (ou du monde) à toute la famille réunie pour l'Épiphanie ou qu'il est le seul à apporter un ordinateur en vacances pour faire quelques traductions quand les autres font la sieste... Si ce sont des connaissances récentes, le traducteur se montrera souvent à l'écoute des autres. Eh oui, car sa grande curiosité fait qu'il a toujours l'air passionné par les gens qu'il rencontre, surtout s'ils ont un métier particulièrement technique, plein de terminologie nouvelle (ah oui, je vous l'ai dit, le traducteur est un geek). II ira même parfois jusqu'à laisser sa carte, on ne sait jamais, la vie est une opportunité de business. Parfois, il ne peut résister à étaler sa culture. Ainsi, si vous passez une soirée entre amis, évitez les quiz et autres Trivial Pursuit ! Après cinq victoires consécutives, plus personne ne veut jouer avec lui.

\section{Avec ses confrères}

Mais là où le traducteur peut être pleinement lui-même, c'est quand il retrouve ses semblables. Vous aurez sûrement du mal à comprendre pourquoi votre conjoint est enchanté de se lever un samedi matin (un samedi matin !) pour assister à une conférence sur la traductologie, l'accord des participes passés en moldave ou le nouveau régime fiscal pour indépendant. Parfois, il accepte même de faire une entorse à la routine bien réglée de ses semaines et prend deux jours pour assister à une conférence internationale de renom sur la convergence entre les métiers de la traduction et les technologies. Entre chercheurs et collègues, le traducteur est dans le bain et peut librement parler d'outils de traduction et comparer ses clients avec ceux de ses collègues. Enfin des gens qui parlent la même langue que lui et le comprennent ! Ces deux jours de colloque auront été fort instructifs pour votre compagnon : il aura appris que dans certaines universités, des chercheurs qui apprécient son utilité et son œuvre travaillent néanmoins à créer des outils qui pourront le remplacer et l'amèneront soit à envisager une nouvelle carrière, soit à s'adapter. Mais votre conjoint est fort et courageux, et ces outils prometteurs ne le découragent pas. Au contraire, il se sent prêt à les étudier et à se les approprier pour en faire non plus des concurrents automatisés, mais de réels outils au service de son travail. Le traducteur connaît la qualité de son travail, maîtrise son art et s'est positionné sur le marché en mettant en avant son talent et ses compétences. Loin d'être technophobe, il a compris qu'une autre utilisation des outils de traduction est possible et qu'elle ne nuira ni à son art, ni à son travail. 
Le soir, en rentrant à la maison, votre cher amour ne cesse de vous raconter cette conférence minute par minute, de vous décrire les personnes qu'il a rencontrées, de vous montrer leurs cartes de visite et de vous rapporter tous ces bons mots et autres jeux d'esprit de traducteurs que vous ne saisissez pas. Soyez sympa et à défaut de comprendre son enthousiasme, faites au moins semblant de vous y intéresser! N'oubliez pas que lorsque vous, vous avez passé une semaine entière avec des collègues que vous ne voudriez surtout pas croiser en plus le weekend, le traducteur, lui, n'a vu personne (sauf sur Twitter et Skype). Comprenez-le, pouvoir retrouver des gens qui vivent comme lui, pour parler d'un sujet qui les intéresse tous (qui a dit qui n'intéresse qu'eux ?), c'est un peu comme aller à Disneyland! Et le plus beau, c'est de voir avec quel enthousiasme il essaiera de vous convaincre de l'importance de la dernière réforme grammaticale ou d'aller voir un film tchèque sous-titré en allemand.

Partenaires de PACS, époux, conjoints, voici donc un aperçu du quotidien de votre cher et tendre, fait de traductions, de culture, de curiosité et très souvent d'humour. Votre traducteur est un passionné et un enthousiaste, n'est-ce pas ce que vous aimez chez lui ?

celine@inotherwords-services.com

Céline PETIT est traductrice indépendante et auteur de l'excellent blog http://www.mavoisinemillionnaire.com/. Elle a spécialement adapté pour Traduire un billet publié sur ce blog. 